\title{
Gestão das ações de segurança alimentar frente à pandemia pela COVID-19
}

\author{
Fábio Resende de Araújo ${ }^{1}$ \\ Dinara Leslye Macedo e Silva Calazans ${ }^{2}$ \\ 1 Universidade Federal do Rio Grande do Norte / Departamento de Administração Pública e Gestão social, Programa de pós- \\ graduação em Gestão Pública, Natal / RN - Brasil \\ 2 Universidade Federal do Rio Grande do Norte / Departamento de Ciências Administrativas, Programa de pós-graduação em \\ Gestão Pública, Natal / RN - Brasil
}

O presente artigo tem como objetivo analisar as estratégias de intervenção adotadas pela gestão pública frente à crise desencadeada pela COVID-19 para redução da insegurança alimentar, baseado em um estudo de caso no Rio Grande do Norte. Com abordagem qualitativa, foram utilizadas a Teoria do Programa e a abordagem dos múltiplos fluxos para análise das ações emergenciais. No estado, a pandemia confluiu para uma janela de oportunidade, evidenciando o papel importante das Políticas Públicas na agenda governamental, como o Programa Restaurante Popular, e favorecendo a elaboração de respostas rápidas e de medidas de adequação na implementação da política. Conclui-se, neste estudo, que esta pode ser considerada uma boa prática e que ela pode servir de referência a outras ações semelhantes pelo país.

Palavras-chave: COVID-19; governos estaduais; políticas sociais; segurança alimentar e nutricional.

\section{Gestión de acciones de seguridad alimentaria frente a la pandemia de COVID-19}

Este artículo tiene como objetivo analizar las estrategias de intervención adoptadas por la administración pública frente a la crisis desencadenada por la COVID-19 para reducir la inseguridad alimentaria, con base en un estudio de caso en Río Grande do Norte. Con un enfoque cualitativo, se utilizaron la teoría del programa y el enfoque de múltiples flujos para el análisis de acciones de emergencia. En el estado, la pandemia se convirtió en una ventana de oportunidad, destacando el importante papel de las políticas públicas en la agenda gubernamental, como el Programa Restaurante Popular, y favoreciendo la elaboración de respuestas rápidas y de medidas de adecuación en la implementación de las políticas. Se concluye que esta puede ser una buena práctica y que puede ser una referencia para otras acciones similares en todo el país.

Palabras clave: COVID-19; gobiernos estatales; políticas sociales; seguridad alimentaria y nutricional.

\section{Management of food security actions during the COVID-19 pandemic}

This article aims to analyze the intervention strategies adopted by public management during the COVID-19 pandemic to reduce food insecurity, based on a case study in the Brazilian state of Rio Grande do Norte. The study used a qualitative approach based on Program Theory and the multiple streams model to analyze the actions. In the state, the pandemic brought a window of opportunity, showing the importance of public policy in the government agenda, such as the program "Restaurante Popular," which offers low-cost meals, and favors quick response and adequacy of means of implementation. The study highlights best practices, inspiring actions all over the country. Keywords: COVID-19; state governments; social politics; food and nutrition security. 


\section{INTRODUÇÃO}

No início do ano de 2020, a Organização Mundial de Saúde declarou à Saúde Pública internacional um alerta emergencial, de mais alto nível, de contaminação pela COVID-19. Devido ao risco em larga escala de disseminação do vírus, foram necessárias ações estratégicas e coordenadas em curto tempo por parte dos governos, tendo em vista a mitigação dos impactos da contaminação (Crodal et al., 2020). Não tardou para que, no mês de março, a COVID-19 fosse caracterizada como uma pandemia, sendo a sua origem e extensão final, assim como a descoberta de vacinas e antivirais, ainda desconhecidas (Chen et al., 2020).

Projeções baseadas nos primeiros casos, pelo Imperial College London (Ferguson, Laydon, \& Nedjati-Gilani, 2020), indicaram que intervenções não farmacêuticas deveriam ser adotadas para reduzir as taxas de contágio das populações, evitando-se, consequentemente, o colapso dos sistemas de saúde. Como recurso interventivo, a prática do isolamento social foi adotada em diversos países, paralisando as atividades não essenciais e vindo a provocar custos sociais e econômicos significativos que afetaram, sobretudo, a população em situação de vulnerabilidade social. Concomitante às medidas de proteção e recuperação da saúde adotadas pela Administração Pública, urgiram decisões estratégicas e alternativas para garantir a segurança alimentar desta população mais vulnerável.

No Brasil, as previsões dos efeitos sociais decorrentes da situação calamitosa, principalmente para os mais vulneráveis economicamente, refletem a profunda desigualdade social no país e destaca o importante papel das políticas públicas de fortalecimento da segurança alimentar como um elemento central nas redes de segurança social. Neste sentido, compreender como programas de acesso a alimentos responderam às pressões impostas pela crise provocada pela COVID-19 na implementação de ações para atendimento da população vulnerável na pandemia se expressa relevante na gestão pública e está sumarizada neste trabalho, por meio de um estudo da operacionalização do Programa do Programa Restaurante Popular (PRP) no Rio Grande do Norte (RN).

Ademais, o PRP, abordado nesta pesquisa, sendo uma política viabilizada por meio de uma terceirização com nove empresas fornecedoras de refeições, sob monitoramento e planejamento estatal, e que ocorre de modo descentralizado em 34 municípios totalizando 56 unidades, o modo de operação deste programa no RN apresenta particularidades para sua implementação, podendo evidenciar um exemplo prático de como articulações entre o público e o privado podem ser fundamentais na superação desse período de agravamento dos determinantes da insegurança alimentar, fomentando aprendizados frente à crise provocada pela COVID-19.

Este estudo objetivou analisar as estratégias de enfrentamento implementadas, em nível estadual, no contexto do Programa Restaurante Popular. Para o alcance deste objetivo geral, buscou-se como objetivos específicos, no contexto do Rio Grande do Norte: 1) compreender o arranjo institucional da rede de Equipamentos Públicos do PRP e 2) analisar a implementação das ações para enfrentamento da COVID-19 no PRP.

Para compreender como as ações se adaptaram frente à pandemia da COVID-19, o programa foi analisado com base nos conceitos da Teoria do Programa na compreensão dos elementos de implementação para análise das ações em uma avaliação in itinere e por meio da abordagem dos fluxos múltiplos de Kingdon (1984). Para análise do arranjo institucional que o programa opera foram destacadas as dimensões planejamento, coordenação, operacionalização e população-alvo. Em cada categoria foram identificados os sujeitos e como estes se organizavam na implementação do programa. 
As ações do programa para o enfretamento da pandemia tiveram como categorias analíticas: problema, planejamento emergencial, prioridades estratégicas, suporte operacional, matriz de ações/causas e monitoramento e avaliação. As evidências empíricas foram extraídas dos decretos normativos ligados ao programa publicados no Diário Oficial do Estado sobre deliberações da Administração pública acerca das medidas de enfrentamento da COVID-19 no âmbito dos restaurantes populares. Para esclarecimentos e elucidação de fatos descritos nos documentos, gestores do nível tático e operacional do Programa foram consultados.

Portanto, este artigo apresenta uma sumarização do cenário de vulnerabilidade social no Brasil, com identificação dos fluxos de problemas, soluções e de pressões políticas da abordagem de Kingdon, e seu agravamento durante à COVID-19, discutindo na sequência a importância das políticas públicas de Segurança Alimentar e Nutricional (SAN) como medida de enfrentamento, sob a perspectiva do Programa Restaurante Popular no Rio Grande do Norte. O estudo destaca as principais ações tomadas pela administração pública e os instrumentos de gestão adotados, assim como os desafios impostos pela pressão temporal de respostas diante da crise pelo novo coronavírus.

\section{A COVID-19 E 0 CENÁRIO DE VULNERABILIDADE SOCIAL NO BRASIL}

No Brasil, a COVID-19 foi caracterizada como emergência nacional em Saúde Pública em fevereiro por meio da Lei n. 13.979/2020, cuja regulamentação e operacionalização se deu pela Portaria n. 356 (2020), a qual trata das medidas de isolamento social e quarentena. O Legislativo Federal (Decreto n. 6, 2020), reconhecendo o estado de calamidade pública no Brasil, requereu que o Estado, baseado na sua infraestrutura e nas características locais, interviesse estrategicamente com ações de enfrentamento à doença nos seus territórios.

Neste contexto, o desafio dos governos é o de articular ações de conciliação conflituosa nos âmbitos da saúde, da economia e das ações sociais (Hafiz, Ring, \& Shntser, 2020). A situação precária da população com renda de subsistência, a mais afetada pela paralisação das atividades, enseja medidas de proteção social imprescindíveis, dentre as quais as relativas à alimentação, objetivadas por este estudo, são uma preocupação imediata.

O Direito Humano à Alimentação Adequada (DHAA) previsto na Constituição Federal Brasileira, em consonância com o Sistema de Segurança Alimentar e Nutricional (SISAN), foi instituído pela Lei 11.346 (2006). A Emenda Constitucional 95 estabeleceu um novo regime fiscal no Brasil, instituindo um teto de despesas primárias que trata da receita corrente líquida nos valores, ajustada apenas pelo índice inflacionário por 20 anos. A medida vem mudando o tamanho do estado, diminuindo o gasto per capita com políticas sociais e repercutindo no financiamento de Políticas Públicas de Segurança Alimentar e Nutricional (SAN) e na efetivação do DHAA (Rossi \& Dweck, 2016; Santarelli, David, Burity, \& Rocha, 2019).

Somam-se a este enfraquecimento da rede de assistência, medidas de austeridade recentes (Reforma Trabalhista de 2017 e Reforma de Previdência de 2019), as quais, apesar de terem como argumentação principal o combate ao desequilíbrio fiscal, resultariam no crescimento econômico, não levaram ao aumento do emprego no país nos últimos anos (Galvão, Castro, Krein, \& Teixeira, 2019; Lobato, Costa, \& Rizzotto, 2019). Ao contrário, tanto o número de desempregados quanto as relações informais de trabalho cresceram (Passos \& Lupatini, 2020).

Em meio ao aumento do desemprego, à diminuição da proteção social, e à potencialização da desigualdade social, surge o coronavírus no Brasil, tornando o planejamento público mais complexo 
frente à crise já em curso. Para Trevisan e Bellen (2008) os gestores públicos têm limitações em tomar decisões e posicionar as instituições estrategicamente ao mesmo tempo de novos acontecimentos, mesmo que a mudança constate para as organizações. Matus (1991), ao abordar o Planejamento Estratégico Situacional, indica que os modos tradicionais de planejamento não consideram a incerteza e a sustentação da governabilidade durante processos de mudança.

A dificuldade é nítida quando se tenta resolver problemas sem explicações claras, em meio a um jogo no qual os governantes não podem assegurar os resultados das suas ações, e dependem de fatores, por natureza, fora de seu controle para solucionar problemas complexos (Matus, 1991). Relacionar a intenção e a realização pode contribuir para uma avaliação da eficácia das ações em relação à complexidade da intervenção (Brousselle, Champagne, Contandriopolos, \& Hartz, 2016; Jannuzzi, 2014). As ações quanto à COVID-19 exigem coordenação de diferentes atores: entes governamentais e não governamentais, governos central e subnacionais, instituições públicas e privadas. Caracterizamse, portanto, pela intersetorialidade e repercutem sistemicamente.

Impulsionada pela crise, a pandemia impõe um reordenamento na agenda governamental, cabendo aos governantes ações decisivas em meio a diferentes fluxos de problemas e alternativas, que conciliem estas possibilidades com interesses políticos diversos em um processo de ajuste mútuo (Capella, 2007; Secchi, 2012).

No Nordeste, o Consórcio Interestadual de Desenvolvimento Sustentável (Consórcio Nordeste), consolidado como um mecanismo de gestão pública, vem a se fortalecer no cenário da COVID-19 pela cooperação entre os governos estaduais e pela adoção de estratégias de enfrentamento comuns baseadas no entendimento da gravidade do momento e no lema "[...] A decisão prioritária é a de cuidar da vida das pessoas" (Carta dos Governadores do Nordeste, 2020, p. 1).

Para que as medidas de distanciamento possam ser aderidas pela população mais pobre se faz necessário priorizar ações proteção social, como forma de atenuar choques financeiros que repercutam na diminuição da renda desses indivíduos. A readaptação e a ampliação de políticas emergenciais de acesso imediato às necessidades básicas, dentre as quais as políticas de SAN nos estados e municípios, impõem-se como uma alternativa capaz de mitigar os efeitos negativos da pandemia.

\section{POLÍTICA PÚBLICA DE SAN COMO MEDIDA DE ENFRENTAMENTO: 0 CASO DO RIO GRANDE DO NORTE}

No Rio Grande do Norte, o Programa Restaurante Popular (PRP), implantado em 2002, é uma dentre as estratégias das Políticas Públicas de combate à pobreza e de garantia do DHAA no estado. A relevância do programa é percebida de modo claro, pois este integra o eixo estratégico do Plano Plurianual (PPA, 2020-2023), figurando nas metas prioritárias no âmbito da Secretaria de Estado do Trabalho, Habitação e Assistência Social (SETHAS) - órgão gestor da Política de Assistência Social no âmbito do Sistema Único de Assistência Social (SUAS). A manutenção da pauta da Segurança Alimentar na agenda governamental do RN indica um protagonismo estadual no exíguo espaço aberto no âmbito federal para a temática.

O PRP integra o SISAN por meio de uma rede de Equipamentos Públicos (EP), que fornece refeições para a população em vulnerabilidade social subsidiadas majoritariamente pelo governo e simbolicamente pela população. Sua operacionalização é descentralizada nos 34 municípios onde atua, com 56 EPs geridos por nove empresas terceirizadas. Cada empresa tem autonomia na compra dos 
seus insumos e na gestão de pessoal dos EPs, conforme as determinações contratuais do Programa. A ação é de responsabilidade da Coordenadoria de Desenvolvimento Social (CODES) em nível central, e tendo as empresas terceirizadas em cada município como corresponsáveis na execução do programa. Cabe a CODES o monitoramento e avaliação da política, que acontece com inspeções às unidades. Quanto ao controle social do PRP, esse é frequentemente exercido pelo Conselho Estadual de Segurança Alimentar e Nutricional (CONSEA) que pauta o programa nas suas reuniões e ações.

O estado do RN, por meio do Decreto Estadual n. 29.524 (2020), dispôs sobre medidas temporárias de distanciamento social para o enfrentamento da situação de emergência provocada pela COVID-19, e proibiu o funcionamento de algumas atividades, como restaurantes. A rede de EPs que serve ao PRP, e atende 42 mil pessoas diariamente, precisou adequar sua estrutura funcional devido aos riscos eminentes de contágio na aplicação da política.

Com a pandemia, questões relativas ao problema da insegurança alimentar vieram a debate, constituindo uma oportunidade de legitimar a importância de programas sociais, bem como a necessidade de fortalecê-los. Kingdon (1984), no modelo dos múltiplos fluxos, indica os momentos de crise como "janelas de oportunidades" que se expressam na convergência de fluxos. Nesse caso, percebemos que os fluxos de problemas (pandemia, desemprego e insegurança alimentar entre os mais pobres), de soluções (necessidade de medidas de isolamento e políticas sociais que diminuam o impacto da crise), e de tensão política (humor nacional pressionando por medidas e grupos de pressão, representados por diferentes atores) convergem para o reconhecimento da relevância do momento.

O PRP, consolidado na agenda pública estadual do RN, adapta-se a essa circunstância ponderando viabilidade técnica, custo tolerável e medidas de proteção à COVID-19 para continuar atendendo seus beneficiários em meio à crise. Medidas foram tomadas em três eixos: a) o deliberativo e decisório, com a deflagração das intenções pela SETHAS/RN por meio de determinações normativas; b) o gerencial, para a elegibilidade da população beneficiária pelo poder público, cujas ações são gerenciadas pela CODES com o apoio do SUAS; e c) o operacional, com a contratação por meio de licitação de empresas privadas especializadas na gestão em alimentação de coletividades, tendo em vista o fornecimento de refeições, com metas específicas de atendimento para cada EP.

O Programa contou com o arranjo institucional existente (Figura 1) e teve ênfase na definição de objetivos prioritários para reduzir a insegurança alimentar da população em vulnerabilidade social nesse período.

Dada a especificidade técnica da produção e distribuição de refeições, a implementação diante do cenário adverso foi possível maior discricionariedade aos gestores. Para definição das prioridades e análise do impacto orçamentário, definiu-se como estratégia: assegurar o acesso, garantir a oferta e manter a qualidade das refeições no Programa durante a crise, considerando as dificuldades práticas e soluções já conhecidas pelos seus operadores. Os gestores, principalmente os do nível tático, tiveram que lidar com a compatibilização entre o recomendado e o possível, pois é nesse nível de ação, na implementação, que segundo Secchi (2012, p. 46) "as intenções são transformadas em ações concretas"

A tendência de aumento da população a ser beneficiada foi discutida, haja vista os efeitos esperados, como a diminuição da renda e o desemprego, contudo, restrições orçamentárias foram impostas. As metas de refeições para cada EP não puderam ser repactuadas com as empresas contratadas devido à natureza burocrática dos contratos licitatórios, tendo sido mantidas as originais. Porém a identificação de pessoas em situação de rua foi redefinida como critério de elegibilidade com isenção do valor da refeição. A assessoria técnica do SUAS articulou-se com associações comunitárias e de controle 
social para o mapeamento e cadastramento destas pessoas nos diversos municípios; não tendo sido possível mensurar antecipadamente a quantidade a ser atendida, foi preciso um sistema de controle e reordenação do recurso público já investido no Programa.

\section{FIGURA $1 \quad$ ARRANJO INSTITUCIONAL DA REDE DE EP DO PROGRAMA DE SAN DO RN}

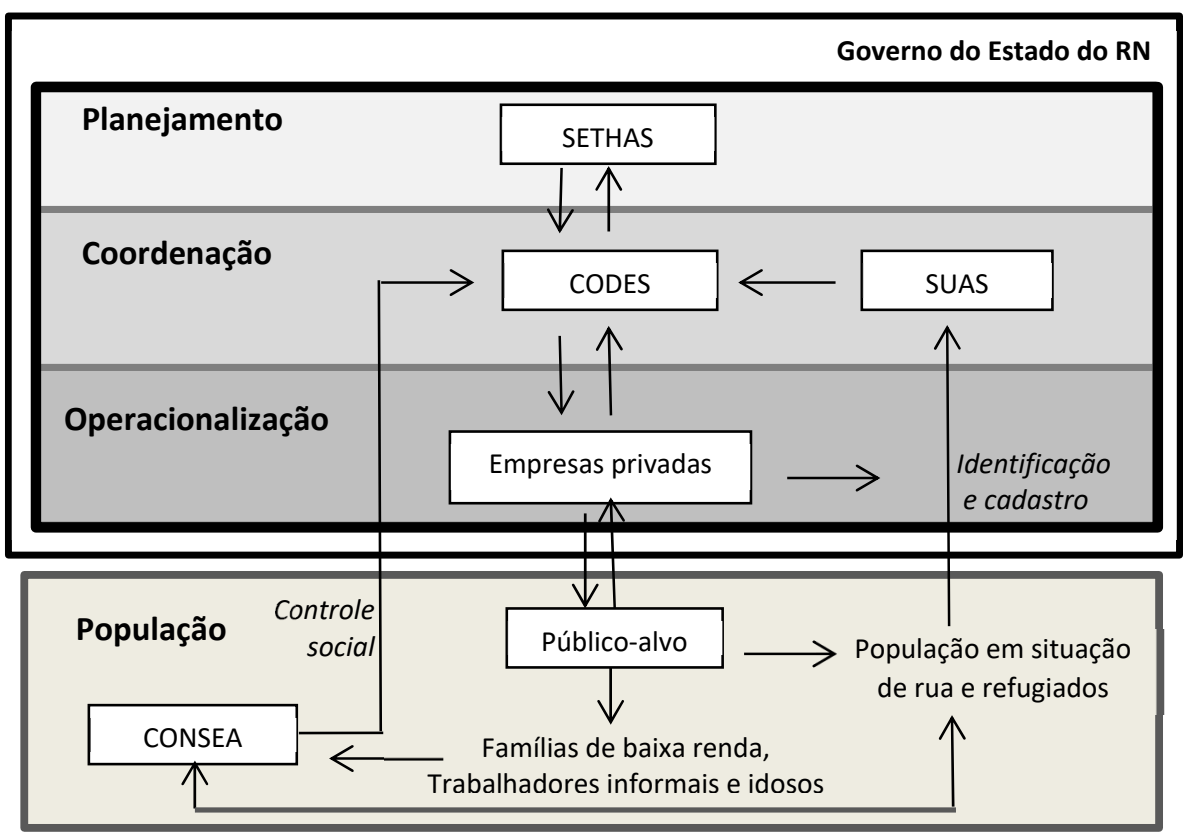

Fonte: Dados da pesquisa (2020).

A CODES considerou o suporte humano, material, financeiro e organizacional necessário para o desenvolvimento das ações a serem cumpridas pelas empresas na gestão local dos EPs durante a mudança da dinâmica operacional do Programa, o que inclui a gestão das informações. Assim, foi estabelecido um plano de comunicações para as diversas partes interessadas (Hafiz et al., 2020; Jannuzzi, 2014), visando dar transparência aos processos e às responsabilidades dos atores envolvidos e contribuir para a adesão das pessoas às medidas necessárias (Crodal et al., 2020; Wilder-Smith \& Freedman, 2020).

Por um lado, as empresas foram notificadas por meio de ofício circular sobre as mudanças necessárias e a atenção ao cumprimento das novas regras (Ofício Circular n. 3/2020/SETHAS). Por outro, os usuários foram comunicados por redes sociais do governo do Estado, com apoio da assessoria de comunicação, além de reportagens nos canais de televisão aberta; a equipe local de cada restaurante divulgou à população as novas condições de atendimento nos EPs, por meio de programas de rádio, carros de som, entre outros. Ao CONSEA foram encaminhadas todas as determinações emitidas pela SETHAS dirigidas aos usuários e às empresas gestoras, a fim de divulgar as informações, apoiar a identificação dos grupos em situação de vulnerabilidade e exercer o controle social para garantia do DHAA. 
Para implementação dos aspectos de natureza operacional, desenvolveu-se uma parceria públicoprivada para além das questões contratuais vigentes, o que exigiu tanto o investimento de recursos privados extraordinários, quanto o envolvimento dos colaboradores e a padronização do serviço oferecido à população pelas nove empresas gestoras com contratos vigentes. A relação de confiança diante da impossibilidade de fiscalização efetiva in loco constituiu um desafio à parte, contando com o apoio das instâncias de controle social. O canal de ouvidoria existente continuou ativo para denúncias e informações, e também foi utilizado para o esclarecimento de dúvidas da população.

Nesse sentido, as decisões tomadas pela Administração Púbica colocaram o processo de "[...] implementação como uma arena política e de relações de troca [...]" (Mota, 2020, p. 138), destacando a importância da coordenação no fluxo das ações de gestão da segurança alimentar frente à COVID-19 (Figura 2).

FIGURA 2 IMPLEMENTAÇÃO DAS AÇÕES PARA ENFRENTAMENTO DA COVID-19 NO PROGRAMA DE SEGURANÇA ALIMENTAR, RIO GRANDE DO NORTE (2020)

Estratégias para enfrentamento da Covid-19 no âmbito dos Programas de Segurança Alimentar no RN

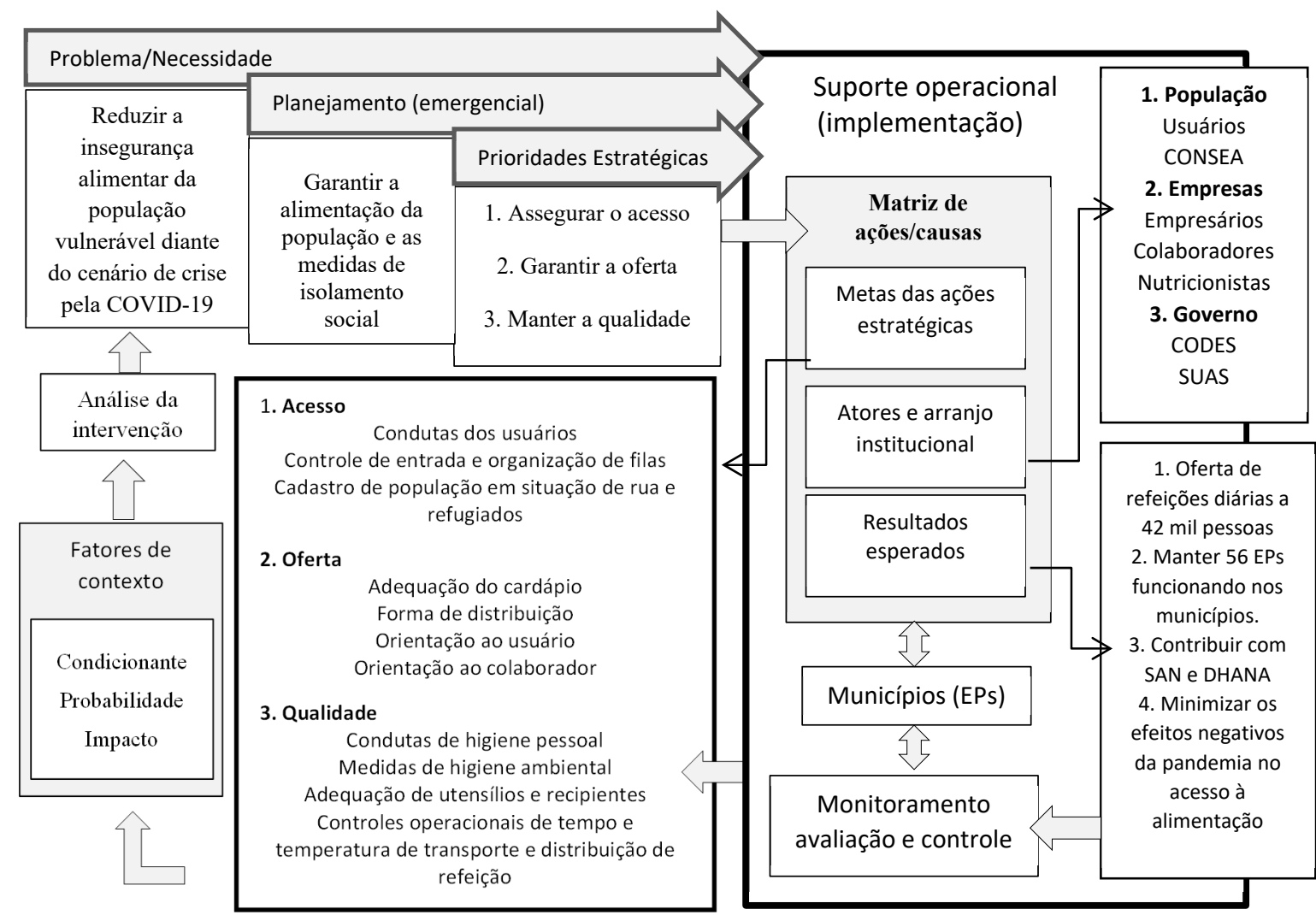

Fonte: Dados da pesquisa (2020). 
Para assegurar o acesso à alimentação, estabeleceu-se a utilização dos próprios EPs em cada município para o fornecimento da refeição, com adequação de horários e aplicação de medidas que minimizassem a propagação do vírus. A orientação presencial dos usuários quanto às medidas de prevenção ficou sob a responsabilidade das empresas (Ofício Circular n, 3/2020/SETHAS).

Em relação à garantia da oferta da alimentação, a forma de distribuição das refeições foi alterada: foi proibida a realização da refeição no restaurante e realizado controle de entrada no seu interior para a distribuição em recipiente apropriado, com consumo em local longe de aglomerações e de circulação de pessoas. Os cardápios foram adaptados para a nova realidade de consumo, optando-se por preparações com menor risco de provocar surtos alimentares. A quantidade adequada da refeição foi definida conforme o planejamento nutricional.

Para manter a qualidade das refeições, sob o aspecto sanitário, foi instituída a adoção de marmita e utensílios descartáveis, conforme orienta Abranches, Oliveira, e Santos (2020) diante da possibilidade de contaminação pelas SARS-CoV-2, decorrente do contato com utensílios e superfícies. Medidas de higiene ambiental e pessoal foram redobradas, destacando-se o cumprimento das condições adequadas de tempo e temperatura no transporte e exposição dos alimentos (RDC n. 216, 2004). Nesse sentido, são obrigatórias a montagem e a distribuição da refeição de forma concomitante, a partir do horário estabelecido para abertura do restaurante.

No monitoramento do Programa a ênfase recaiu sobre a necessidade de ações corretivas em direção às metas estabelecidas, o que se revela adequado para resultados de curto prazo (Jannuzzi, 2014). Os critérios de desempenho, importantes para o cumprimento das avenças pactuadas em contratos de serviços terceirizados na Administração Pública (Marinho, Andrade, Marinho, \& Mota, 2018), normalmente utilizados, ficaram restritos ao acompanhamento das medidas emergenciais impostas pela circunstância e dos produtos esperados da ação: manutenção dos EPs abertos ao público-alvo com alcance da meta de refeições diárias. Sendo assim, devem ser consideradas possíveis situações de invalidação das ações programadas (Matus, 1991) que podem conduzir a contextos de inconsistências, insuficiências ou desperdícios no decurso da ação de cada meta.

O real acesso da população com isenção do valor da refeição pode ter adicionado interesses privados, com superdimensionamento em detrimento do pouco nível de controle, não sendo possível verificar ainda seu impacto no cumprimento das legislações fiscal e orçamentária. Não é possível desconsiderar o aumento do custo operacional decorrente, principalmente, de gastos extras com descartáveis, por parte das empresas, o que pode exercer alguma pressão no funcionamento adequado dos EPs.

Considera-se que os efeitos negativos da ausência da fiscalização sistemática, apesar de impacto relevante, sejam de baixa probabilidade de ocorrência, e que a possibilidade de encerramento de contratos, aplicação de penalidades ou fechamento dos restaurantes possam exercer alguma coerção sobre as empresas fornecedoras (Casula, 2017). De fato, as normas elaboradas exerceram um poder regulatório sobre o comportamento das empresas fornecedoras, contribuindo para a boa governança do empreendimento como um todo.

É possível que as medidas de distanciamento para evitar aglomerações não sejam suficientemente conduzidas pelas equipes locais. Nesse caso, intensificam-se os atos normativos direcionados às empresas e se reforçam os meios de comunicação, sensibilização e colaboração necessários por parte dos usuários.

Em relação ao alcance das metas de qualidade, foram consideradas suficientes as mudanças prévias no cardápio, no transporte da refeição e nas orientações para o seu consumo posterior. Outrossim, em caso de algum colaborador apresentar sintomas de contaminação, ou estabelecer contato com 
algum caso suspeito, são previstas providências, por parte da empresa, para a substituição do agente (Abranches et al., 2020), mantendo-se a equipe com o número suficiente de pessoas para atuar na produção e no fornecimento das refeições.

Conclui-se que todas as ações emergenciais exigem uma estratégia de relacionamento continua entre os âmbitos público e privado e requerem apoio por parte dos investidores privados às condutas de mitigação dos efeitos negativos da situação atual.

\section{CONSIDERAÇÕES FINAIS}

A intervenção realizada pelo estado do Rio Grande do Norte para enfrentamento da COVID-19, por meio de Programa de SAN, numa perspectiva "em ação", contribui com a assertividade dos instrumentos de gestão utilizados, produzindo uma eficácia condizente com a escala e a complexidade das estratégias priorizadas diante dos desafios operacionais impostos pela execução intempestiva.

Favorece o cenário da ação a capacidade estrutural interiorizada do PRP nos municípios e de interlocução dos atores, já consolidada antes da crise, dando agilidade ao processo burocrático estadual envolvido.

Destaca ainda, paradoxalmente, um aspecto positivo da pandemia como janela de oportunidade para manutenção da pauta, de elevada importância, dos Programas de acesso a alimentos desenvolvidos pelo setor público, fomentando debates políticos e acadêmicos necessários para a legitimidade das políticas sociais no Brasil e incentivando organizações da iniciativa privada a adotarem medidas conjuntas com o setor público. Quiçá, esta experiência que serviu de objeto para este estudo possa servir como referência a outras ações semelhantes no país. 


\section{REFERÊNCIAS}

Abranches, M. V., Oliveira, C. T., \& Santos, V. S. (2020). COVID -19: Estratégias para adaptação da produção, comercialização e gestão de estabelecimentos do setor de alimentos. São Carlos, SP: Scienza.

Brousselle, A., Champagne, F., Contandriopoulos, A. \& Hartz, Z. (Orgs). (2016). Avaliação: conceitos e métodos. Rio de Janeiro, RJ: FIOCRUZ.

Capella, A. C. N. (2007). Perspectivas teóricas sobre o processo de formulação de políticas públicas. In G. Hochman, M. Arretche, \& E. Marques. (Orgs.), Políticas Públicas no Brasil. Rio de Janeiro, RJ: FIOCRUZ.

Carta dos Governadores do Nordeste. (2020, 25 de março). Consórcio Interestadual de Desenvolvimento do Nordeste. Salvador, BA. Recuperado de https:// agorarn.com.br/nacionais/governadores-donordeste-vao-manter-medidas-de-isolamento/

Casula, M. (2017). Quem governa na governança (local)? Reflexões teóricas e evidências empíricas. Revista de Administração Pública, 51(6), 1121-1138. Recuperado de https://doi. org/10.1590/0034-7612161618

Chen, P., Mao, L., Nassis, G. P., Harmer P., Ainsworth, B. E., \& Fuzhong L. (2020). Coronavirus disease (COVID-19): the need to maintain regular physical activity while taking precautions. Journal of Sport and Health Science, 9(2), 103-104.

Crodal, J., Oliveira, W. K., Frutuoso, R. L., Mandetta, L. H., Baia-da-Silva, D. C., Brito-Sousa, J. D. ... Lacerda, M. V. G. (2020, abril). COVID-19 in Brazil: advantages of a socialized unified health system and preparation to contain cases. Revista da Sociedade Brasileira de Medicina Tropical, 53(e20200167). Recuperado de https://doi.org/10.1590/0037-86820167-2020

Decreto Legislativo n. 6, de 2020. (2020). Reconhece, para os fins do art. 65 da Lei Complementar n. 101, de 4 de maio de 2000, a ocorrência do estado de calamidade pública, nos termos da solicitação do Presidente da República encaminhada por meio da Mensagem no 93, de 18 de março de 2020. BrasíliaDF. Recuperado de http://www.planalto.gov.br/ ccivil_03/portaria/DLG6-2020.htm

Decreto n. 29524 de 17 de março de 2020. (2020). Publicado em 18 de março de 2020. Dispõe sobre medidas temporárias para o enfrentamento da Situação de Emergência em Saúde Pública provocada pelo novo Coronavírus (COVID-19). Rio Grande do Norte. DOE - RN.

Ferguson, N. M., Laydon, D., Nedjati-Gilani, G., Natsuko, I., Kylie A., Marc, B., ... Ghani, C. A. (2020, 16 de março). Impact of non-pharmaceutical interventions (NPIs) to reduce COVID-19 mortality and healthcare demand. London, UK: Imperial College London. Recuperado de https://www. imperial.ac.uk/media/imperial-college/medicine/ sph/ide/gida-fellowships/Imperial-CollegeCOVID19-NPI-modelling-16-03-2020.pdf

Galvão, A., Castro, B., Krein, J.D., \& Teixeira, M. O. (2019). Reforma Trabalhista: precarização do trabalho e os desafios para o sindicalismo. Cadernos CRH, 32(86), 253-270. Recuperado de https://doi. org/10.9771/ccrh.v32i86.30691

Hafiz H., Oie S., Ring, D. M., \& Shntser, N. (2020). Regulating in pandemic: evaluating economic and financial policy responses to the coronavirus crisis. Boston College Law School Legal Studies (Research Paper n. 527). Recuperado de https://papers.ssrn. com/sol3/papers.cfm?abstract_id $=3555980$

Jannuzzi P. M. (2014, maio-agosto). Avaliação de programas sociais: conceitos e referenciais de quem a realiza. Estudos em Avaliação Educacional, 25(58), 22-42.

Kingdon, J. (1984). Agendas, Alternatives, and Public Policies. Boston, USA: Little, Brown.

Lei $n$. 11.346, de 15 de setembro de 2006. (2006). Cria o Sistema Nacional de Segurança Alimentar e Nutricional - SISAN com vistas em assegurar o direito humano à alimentação adequada e dá outras providências. Brasília-DF. Recuperado em http://www.planalto.gov.br/ccivil_03/_Ato20042006/2006/Lei/L11346.htm

Lei n. 13.979, de 6 de fevereiro de 2020. (2020). Dispõe sobre as medidas para enfrentamento da emergência de saúde pública de importância internacional decorrente do coronavírus responsável pelo surto de 2019. Brasília-DF. Recuperado de http://www. planalto.gov.br/ccivil_03/_ato2019-2022/2020/lei/ L13979.htm

Lobato, L. V. C., Costa, A. M., \& Rizzotto, M. L. F. (2019). Reforma da Previdência: o golpe fatal 
na seguridade social brasileira. Revista Saúde em Debate, 43(120), 5-14.

Marinho, R. C. P., Andrade, E. P., Marinho, C. R. P., \& Motta, E. F. R. O. (2018). Fiscalização de contratos de serviços terceirizados: desafios para a universidade pública. Gestão \& Produção, 25(3), 444457. Recuperado de https://doi.org/10.1590/0104530x1595-18

Mota, L. F. (2020). Estudos de implementação de políticas públicas: uma revisão de literatura. Sociologia, Problemas e Práticas, 92, 133-150. Recuperado de https://dx.doi.org/10.7458/ SPP20209211728

Ofício Circular no 3/2020/SETHAS de 18 de março de 2020. (2020). CODES/SETHAS - Chefia de Gabinete/SETHAS - Secretaria-SETHAS. Secretaria Estadual do Trabalho, Habitação e Assistência Social. Rio Grande do Norte.

Passos, S. S., \& Lupatini, M. (2020). A contrarreforma trabalhista e a precarização das relações de trabalho no Brasil. Revista Katálysis, 23(1), 132-142. Recuperado de https://dx.doi.org/10.1590/198202592020v23n1p132

Resolução RDC n. 216, de 15 de setembro de 2004 da Agência Nacional de Vigilância Sanitária (ANVISA). (2004). Dispõe sobre Regulamento Técnico de Boas
Práticas para Serviços de Alimentação. Diário Oficial da União (DOU), Brasília/DF.

Rossi, P., \& Dweck, E. (2016). Impactos do novo regime fiscal na saúde e educação. Cadernos de Saúde Pública, 32(12). Recuperado de https://dx.doi. org/10.1590/0102-311x00194316

Santarelli, M., David, G., Burity V. \& Rocha, N. C. (2019). Informe Dhana 2019: autoritarismo, negação de direitos e fome. Brasília, DF: FIAN Brasil. Recuperado de https://fianbrasil.org.br/informedhana-2019-faca-download-aqui/

Secchi, L. (2012). Políticas Públicas: conceitos, esquemas de análises, casos práticos. (pp. 33-60). São Paulo, SP: Cengage Learning.

Trevisan, A. P., \& Van Bellen, H. M. (2008). Avaliação de políticas públicas: uma revisão teórica de um campo em construção. Revista de Administração Pública, 42(3), 529-550. Recuperado de https://doi. org/10.1590/S0034-76122008000300005

Wilder-Smith, A., \& Freedman, D.O. (2020). Isolation, quarantine, social distancing and community containment: pivotal role for old-style public health measures in the novel coronavirus (2019-nCoV) outbreak. Journal of Travel Medicine, 13(27), 1-4.

\section{Fábio Resende de Araújo}

https://orcid.org/0000-0001-8268-0078

Doutor em Administração e professor adjunto do Departamento de Administração Pública e Gestão Social e do Programa de pós-graduação em Gestão Pública da UFRN. E-mail: fabio.resende@ufrn.edu.br

\section{Dinara Leslye Macedo e Silva Calazans}

https://orcid.org/0000-0003-4884-8599

Doutora em Administração e professora adjunta do Departamento de Ciências Administrativas e do Programa de pós-graduação em Gestão Pública da UFRN. E-mail: dinaraleslye@yahoo.com.br 